\title{
GPPS-BJ-2019-182
}

\section{OPTIMIZED DESIGN OF THE VOLUTE BETWEEN TURBINE AND RECUPERATOR IN MICRO GAS TURBINE}

\author{
Li Yaoyang \\ 1. Institute of Engineering Thermophysics, \\ Chinese Academy of Sciences \\ 2. University of Chinese Academy of Sciences \\ liyaoyang@iet.cn \\ Beijing, China
}

Institute of Engineering

Thermophysics, Chinese

Academy of Sciences

yinzhao@iet.cn

Beijing, China

\author{
Wang shaolin \\ Institute of Engineering \\ Thermophysics, Chinese \\ Academy of Sciences \\ wangshaolin@iet.cn \\ Beijing, China
}

\author{
Zhang hualiang \\ 1. Institute of Engineering Thermophysics, \\ Chinese Academy of Sciences \\ 2. University of Chinese Academy of Sciences \\ zhanghl@iet.cn \\ Beijing, China
}

\section{ABSTRACT}

In order to improve the aerodynamic performance of the inlet volute of the micro gas turbine recuperator, according to the goal of airflow loss and evenness, designing a two steps volute profile and optimizing it to obtain the volute profile with the smallest aerodynamic lose. On this basis, add perforated sheet to make the airflow more uniform and study the effect of the angle and position of the perforated sheet on the uniformity of the flow. The research shows that the two steps volute design can greatly increase the uniformity of the airflow without changing the loss characteristics; the loss coefficient is reduced by $11.1 \%$ in the design condition with optimization; the fluid movement at high radii upstream of the perforated sheet is mainly determined by Coanda effect and space blocking effect. The Coanda effect causes the fluid attaching the wall to a high radius and increase the flow velocity in it. The space blocking effect caused by the small space makes the fluid moving toward the lower radius and thus increase the flow velocity in it. The best axial position is obtained which the two effect phases are balanced. Carry out parametric design to form design criteria to guide the design of this type of volute.

\section{INTRODUCTION}

The micro gas turbine has the advantages of compact structure, high reliability and simple maintenance. It's widely used in the fields of vehicle, ship power and distributed power generation[1]. In order to improve the efficiency and power-to-weight ratio, a compact annular recuperator can be designed for surrounding the micro gas turbine. As shown in Figure 1, when the airflow leaves the turbine, it turns 180 deg and then enters the recuperator. However, the flow angle is so large that causing the big aerodynamic loss. The fluid attached to the outer wall causes the nonuniform velocity in the recuperator inlet, which greatly reduces the efficiency of recuperator. Therefore, it's necessary to optimize the volute between turbine and recuperator.

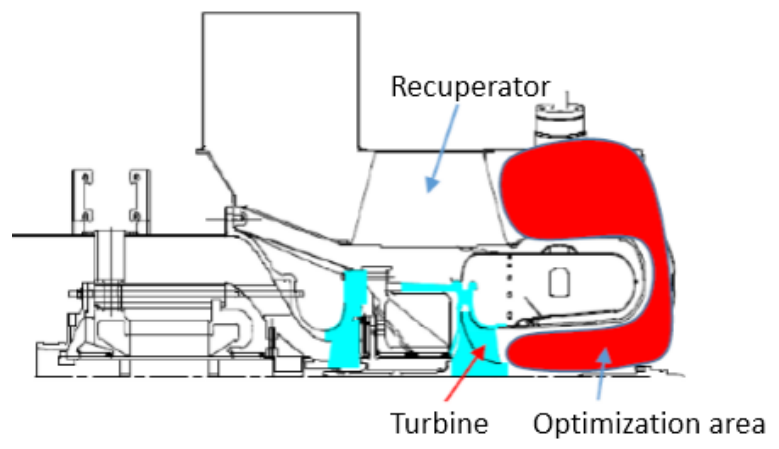

Figure 1 Sketch maps of the volute in micro gas turbine

Since the profile of the volute has an important influence on the overall performance of turbines, lots of research has been done on design of the volute [2]. The traditional design methods mainly include the constant circulation method, the average speed method, and the structural square method. However, the above methods are designed under some hypothesis, and can not truly reflect the internal flow of the volute, so there is still room for optimization. Li Yan [5] 
optimized the centrifugal fan volute by changing the helical angle, and used the quadratic orthogonal regression experimental design method to generate variable database. Calculating that and select the optimal result for each variable combination. Further, Chen Zonghua [6] used the radial basis function neural network as the surrogate model to establish a approximate empirical relation between geometry and aerodynamic loss for the centrifugal compressor inlet section, and then used the neural network algorithm to optimize the volute profile, which increased the stage efficiency by $1.26 \%$ at the design point. After that Huang Ende [7] established the automation optimization platform for Numeca and Isight, and also used the surrogate model to optimize non-axisymmetric exhaust hood, which reduced the total pressure loss of by $9.82 \%$. However, the geometry of the volute involved in the study above is different from this paper. There are few studies on the form of the axisymmetric volute between turbine and recuperator which turn around $180 \mathrm{deg}$. In engineering, the volute of Capstone's regenerative gas turbine is similar to this paper and adopts a segmented diffuser structure design, which greatly improves the recuperator effectiveness and overall efficiency, but the design principle has not been reported.

In terms of airflow uniformity, perforated sheet is usually used in engineering to imp rove the flow distribution. Wang Xiaoyi et al [8] use the air guide plate and baffle to achieve airflow rectification in the wind tunnel inlet. Shao Dong et al [9] designed the radial air intake system, adding a honeycomb structure inside the flow channel to make the airflow more uniform than before. XueleiZhang et al. [10] added a perforated plate to the air-cooled steam condenser to rectify the airflow to achieve a more uniform flow field distribution. Wu Hao [11] used perforated sheet in the inlet section of shell-and-tube heat exchanger. Due to the improved flow distribution, the heat transfer efficiency much better than that of the heat exchanger without perforated sheet. From the above, the perforated sheet is very effective to improve the uniformity of air flow in each device. When used computational fluid dynamics (CFD) to solve it, questiones come that it is difficulty to generate the mesh of the perforated sheet, more scholars use the porous medium model [12] to simulate it. However, the above studies mainly show that the perforated sheet can improve the uniformity of the air flow, and less research on the details of the placement angle and position of the perforated sheet.

However, there are few papers researched about how to improve the aerodynamic performance of the inlet volute of the micro gas turbine recuperator according to the goal of airflow loss and evenness. This paper puts forward the idea of two steps profile design method and researches the angle and position of the perforated sheet to understand the flow mechanism and form design criteria to guide the design of this type of volute. Section 2 provides brief introduction about geometric model and numerical method. Section 3 introduces the idea of two steps profile design method and optimal Latin hypercube[13] design experimentis method. It need using CFD method to calculate it and obtain the profile with the smallest aerodynamic loss. On the basis of above,
Section 3 also introduces the perforated sheet which was added to make airflow more uniform, and the influence of the angle and position of the perforated sheet on the uniformity of the airflow was studied. The conclusions are summarized in section 4 .

\section{GEOMETRIC MODEL AND NUMERICAL METHOD}

\subsection{Geometric model}

In this study, the position of volute is decided by turbine outlet and recuperator inlet which corresponding to volute inlet and outlet. Airflow enters volute from a low radius and flows out at a high radius. The section of turn around is designed with a simple circular arc.The 2-D geometric model is show in Fig.2 and its 3-D structure is revolve around axial.

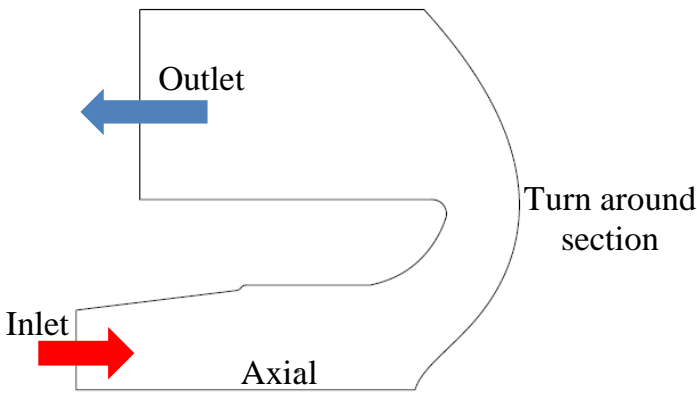

Figure 2 2-D geometric model for original volute

\subsection{Numerical method}

The numerical calculation is solved by ANSYS Fluent, and the two-dimensional axisymmetric model is selected. All calculations adopt the S-A turbulence model. For the perforated sheet, using Fluent porous medium model to replace it. The boundary conditions are given the mass flow and total pressure, and the outlet is given static pressure. The detailed parameters are shown in the table 1. Using ICEM to divide the two-dimensional surface mesh.

Table 1 Boundary conditions

\begin{tabular}{cc}
\hline Boundary & Boundary conditions \\
\hline Inlet & Mass flow: $m=0.95 \mathrm{~kg} / \mathrm{s}$ \\
& Total temperature: $T_{\text {in }}^{*}=760 \mathrm{~K}$ \\
Outlet & Pressure: $p_{\text {out }}=100 \mathrm{KPa}$ \\
\hline
\end{tabular}

The grid-independent verification is done to ensure the accuracy of numerical calculation. As shown in Fig.3, the pressure drop represents the pressure different between inlet and outlet, it's one of the most critical parameters in this article. When the number of meshes reaches 18 000, the calculation results basically do not change with the number of grids. In addition, literature [16] also using axisymmetric model and the geometric structure is similar with this paper. The results of calculation in this paper are similar to the literature. 


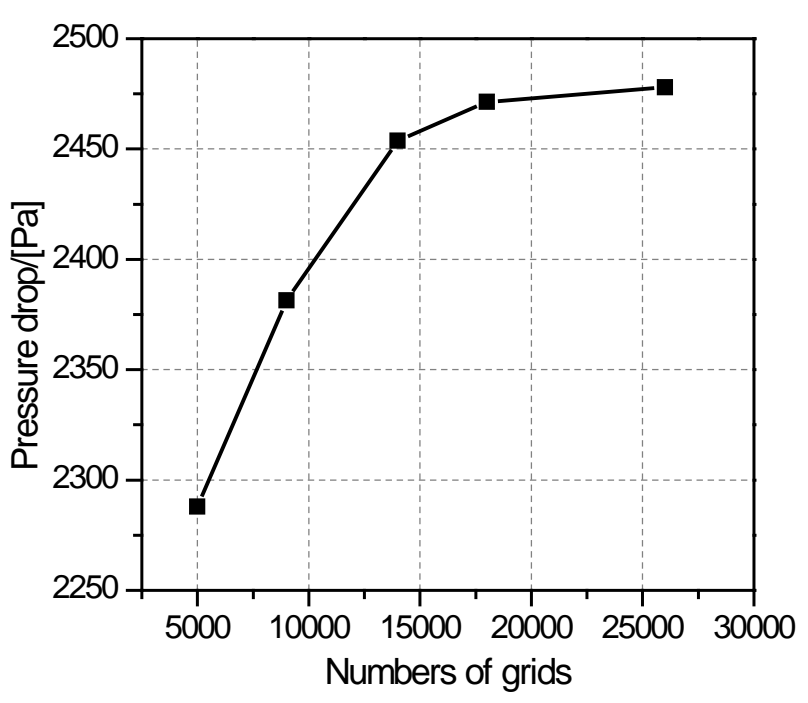

Figure 3 Grid independence check

\section{OPTIMIZATION DESIGN}

\subsection{Optimization indicator}

The optimization index of the volute mainly includes aerodynamic loss and uniformity of the outlet airflow.

\subsubsection{Loss coefficient}

The loss coefficient is defined as follows:

$$
\sigma=\frac{p_{i n}^{*}-p_{\text {out }}^{*}}{0.5 \rho_{\text {in }} v_{\text {in }}^{2}}
$$

Where $P_{\text {in }}^{*}$ and $P_{\text {out }}^{*}$ respectively indicate the total pressure of inlet and outlet, $\rho_{\text {in }}$ and $v_{\text {in }}$ indicate density and velocity of volute inlet. It follow that the $\sigma$ is small when the aerodynamic loss is small.

\subsubsection{Total pressure recovery coefficient}

Total pressure recovery coefficient is defined as follows:

$$
p_{\text {rec }}=\frac{p^{*}}{p_{\text {in }}^{*}}
$$

Where $P^{*}$, the total pressure at any section.

\subsubsection{Unevenness coefficient}

Define airflow unevenness coefficient as follows:

$$
\gamma=\frac{\bar{V}_{\text {max }}-\bar{V}_{\text {min }}}{\bar{V}_{\text {out }}}
$$

Where $\bar{V}_{\text {out }}$,the area average velocity of the outlet. $\bar{V}_{\max }$, is the area average velocity which bigger than $\bar{V}_{\text {out }}$. $\bar{V}_{\text {min }}$, the area average velocity which less than $\bar{V}_{\text {out }}$.

\subsection{Two steps volute design}

The internal flow field is carried out by CFD method. The streamline is shown in figure 4 and the axial velocity in outlet is shown in figure 6 . It can be seen that under the turn around effect, the fluid flow along the outer wall surface, so that the velocity near the outer wall surface is the largest, while the velocity at other locations is small, and the airflow velocity is seriously uneven, which greatly affects the heat exchange effect of the recuperator.

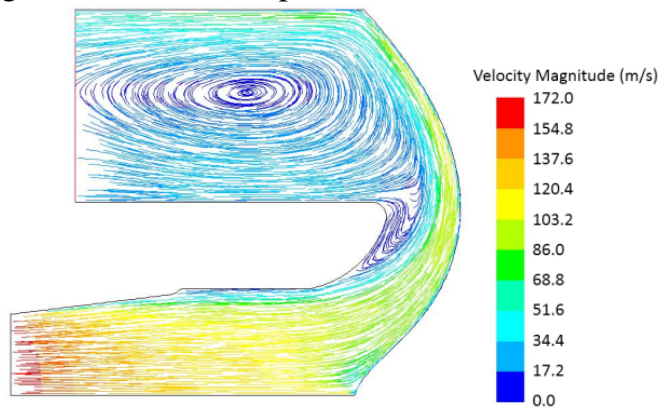

Figure 4 Streamline for original volute

In view of this, a design idea of two steps volute using the Coanda effect[14] and flow separation conditions is proposed. The airflow leaves volute in the middle of outlet and is guided by the profile, as show in figure 5 . Figure 6 shows the dimensionless axial velocity in outlet for different volutes, and the axial velocity is dimensionless with inlet velocity. The results show that with the two steps design, the uniformity of the outlet flow is greatly improved without affecting the flow loss.

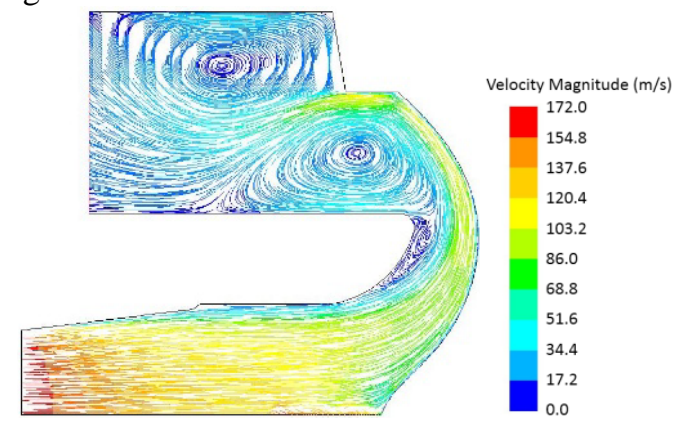

Figure 5 Geometry and streamline for two steps volute

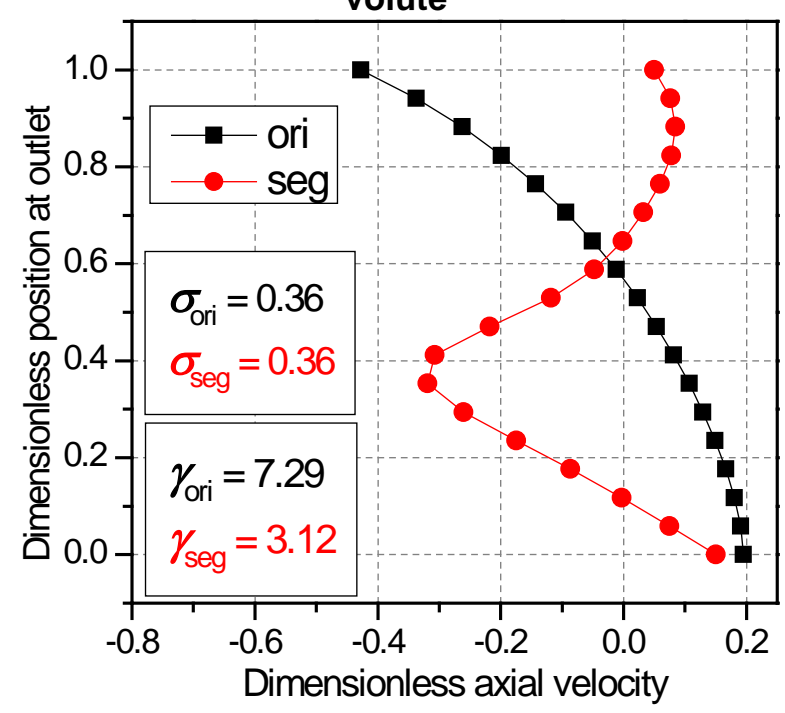

Figure 6 Axial velocity along outlet for original and two steps volutes 


\subsection{DOE-based turn around profile optimization}

\subsubsection{Parameterization}

The research on the exhaust volute shows that the turn around section has a great influence on the overall aerodynamic loss. Therefore, the parametric model of the turn around section is shown in this paper, and it is shown by the red line in figure 7 . Since the Bezier curve is highly steerable, the overall curvature can be guaranteed to be smooth and easy to control [15]. Therefore, the 5 point 4th order Bezier curve is used to fit the curve of the turn around section, and $\mathrm{x} 1, \theta 1, \mathrm{y} 1, \theta 2, \mathrm{y} 2$ are selected as variables. Where $\mathrm{x} 1$ represents the degree of airflow deflection, $\theta 1$ and $\mathrm{y} 1$ represent the degree of contraction of the airflow before passing through the throat, and $\theta 2$ and y2 represent the degree of expansion of the airflow after passing through the throat. Each variable changes the wall profile, thereby affecting the aerodynamic performance.

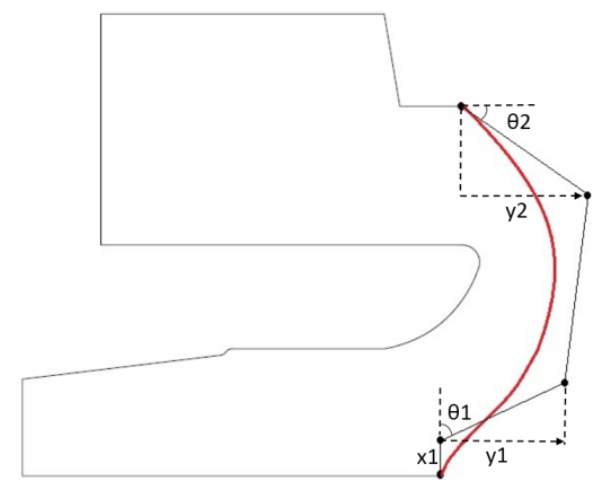

Figure 7 Parameterization for turn around section profile

\subsubsection{Design of experiments}

The values of the variables in the above-mentioned profile volute are used as reference values, and each variable is floated up to $10 \%$. Optimal Latin hypercube can make the test data sufficiently uniform. Using it to design experimentis and generate 100 sets of variable combinations and each variable combination corresponds to a volute profile. Solving $\mathrm{N}-\mathrm{S}$ equation by ANSYS Fluent for each volute profile.

\subsubsection{Optimization results}

The volute profile with the smallest loss coefficient is selected as the optimal profile. As shown in the figure 8, the red profile represents two step volute while blue represents the optimized profile. At the same time, the loss coefficient of two volutes for different massflow rates is show in figure 9. It shows that the optimized profile has a reduced loss in the massflow range of 0.8 to $1.3 \mathrm{~kg} / \mathrm{s}$.

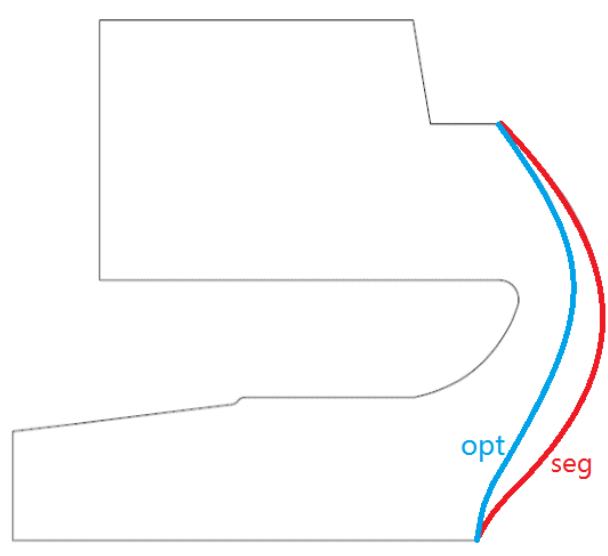

Figure 8 Comparison of volute profiles with and without optimization

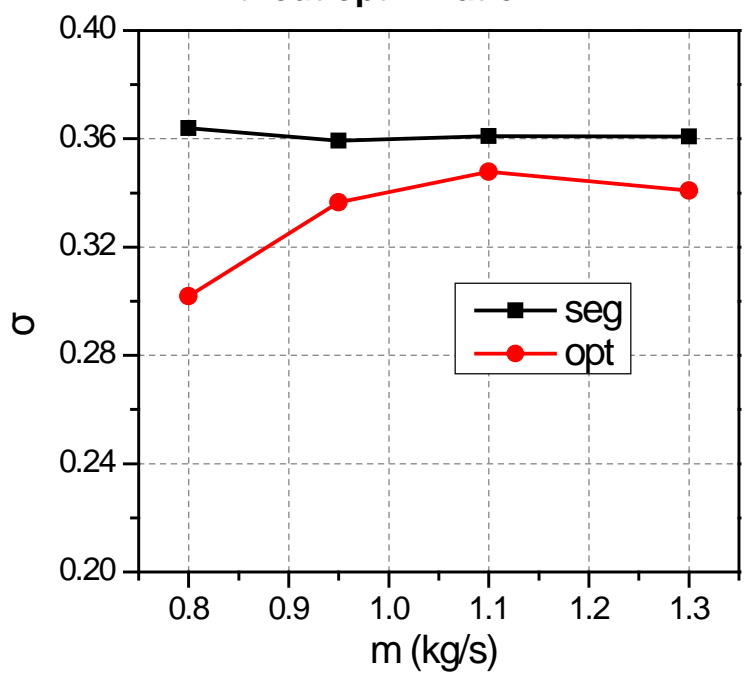

Figure 9 Comparison of loss coefficient with and without optimization

Figure 10 shows the total pressure recovery coefficient for different cut planes along the flow direction. It can be seen that the difference between two volutes is biggest in 3 sections. This indicates the aerodynamic loss for volute without optimizate mainly caused between 2 and 3 sections, which is the turn around section, further research can be focused on this section.

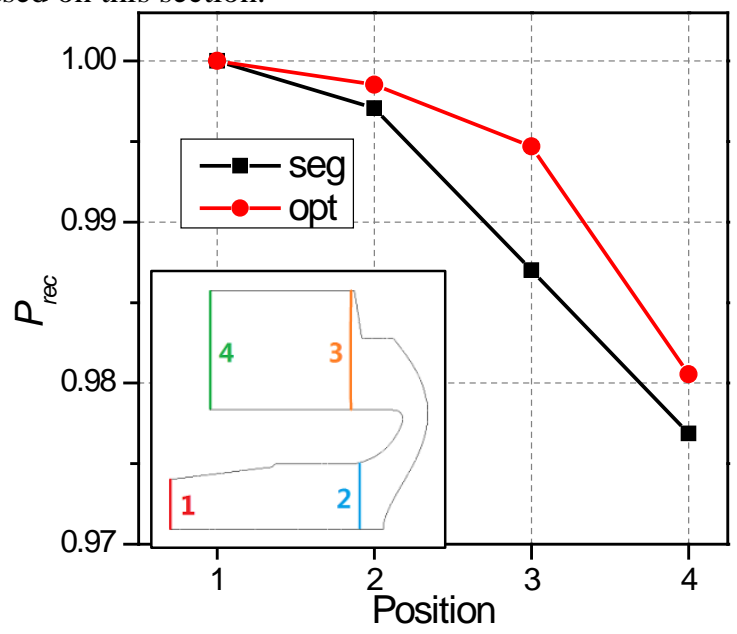

Figure 10 Total pressure recovery coefficient for different sections 
Figure 11 shows the internal flow field of the volute turn around section with and without optimization. It can be seen that the optimized profile is more conformable to the airflow, the recirculation zone at the turning around section is reduced, the vortex disappears obviously, and the loss is reduced. (a) seg

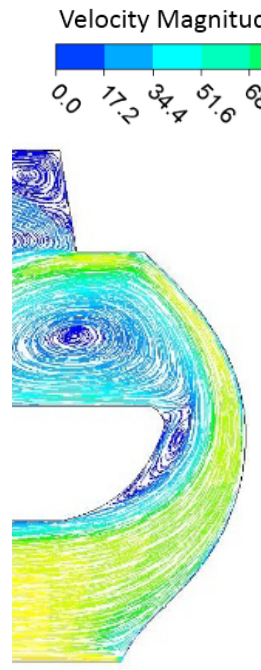

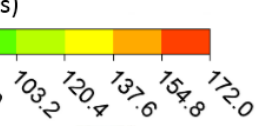

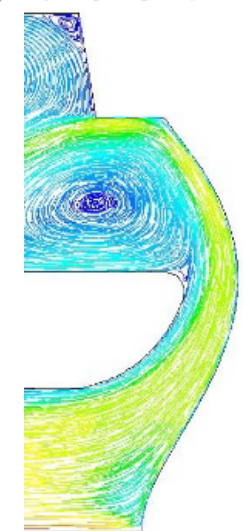

(b) opt
Figure 11 Comparison of volute streamline with and without optimization

\subsection{Perforated sheet optimization}

Though two steps volute design can improve the airflow evenness, it can not meet the engineering requirements, only through the volute profile can not achieved it. Therefore, based on the above research, the perforated sheet is added to the optimal volute to increase the uniformity of the outlet flow. The porous media model in the Fluent software was used to simulate the perforated sheet to reduce the difficulty of meshing, and the influence of the angle and position of the perforated sheet on the uniformity of the airflow was studied.

\subsubsection{Influence of perforated sheet angle}

In order to investigate the influence of the installation angle of the perforated sheet on the uniformity of the airflow, the vertical, right obilique and lift obilique perforated sheet were studied. The geometry of different perforated sheet is shown in the figure 12 .

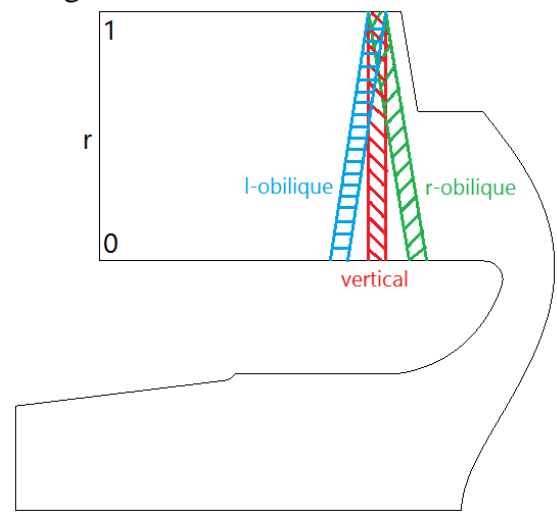

Figure 12 Geometry for different perforated sheet angle
Figure 13 shows the dimensionless axial velocity at the exit of the volute with different angle of perforated sheet, where the red line, blue line and green line represents the vertical, left obilique and right obilique perforated sheet volute respectively. It can be seen that the aerodynamic losses of the different volute are basically the same. The evenness of the flow of the vertical perforated sheet and the left inclined perforated sheet volute is basically similar, but the right inclined perforated sheet volute is poor. In addition, the loss is increased a lot due to the introduction of perforated sheet.

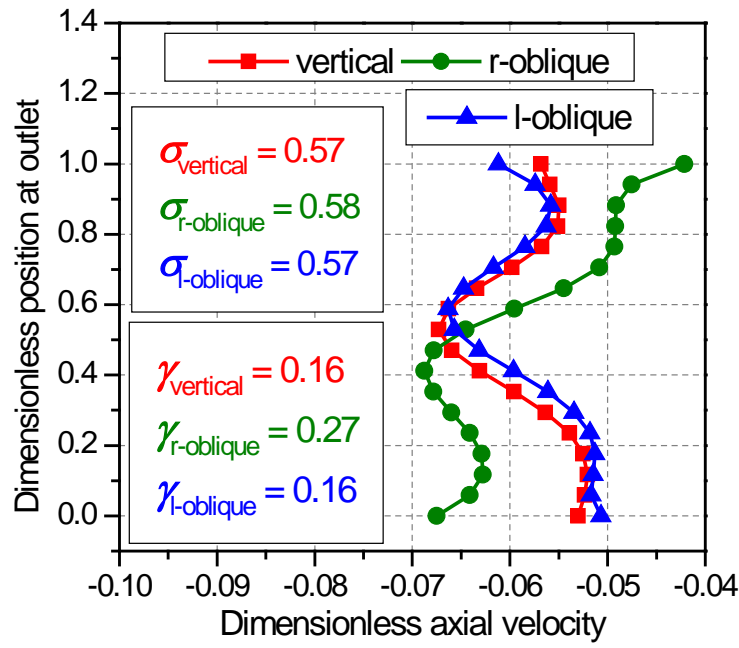

Figure 13 Axial velocity distribution at volute outlet for different perforated sheet angle

To explore the cause of the difference in airflow uniformity, draw streamline diagram of different volutes to analysis, as shown in figure 14 . For the volute of the right inclined perforated sheet, the perforated sheet is too close to the upstream boundary in a high radius to restrain the airflow flow to a high radius, so that the gas flow velocity is small in high radius. The volute of the left inclined and the vertical perforated sheet is not limited by the space blocking effect due to the large gap upstream of the perforated sheet. Therefore, when the space blocking effect is not considered, the perforated sheet angle has less influence on the airflow uniformity and aerodynamic loss.

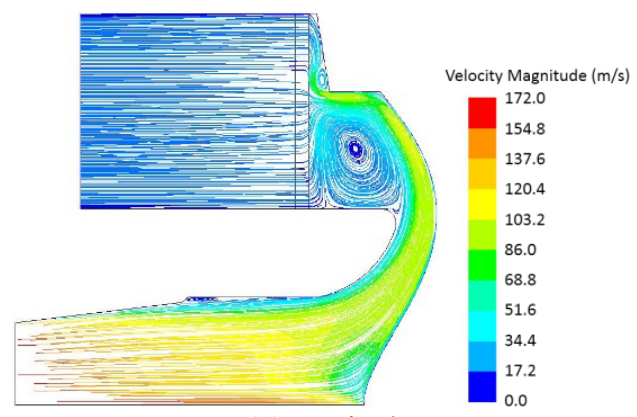

(a) vertical 


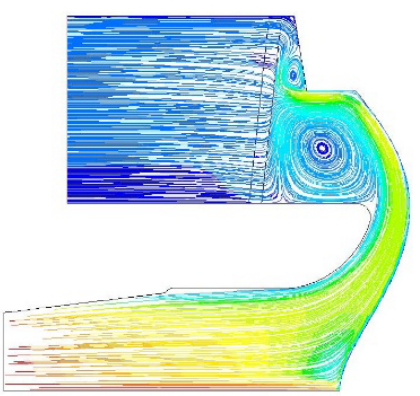

(b) l-oblique

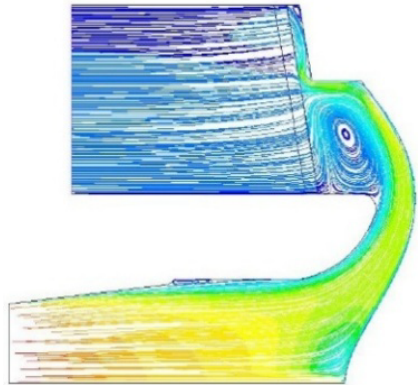

(c) r-oblique
Figure 14 Comparison of volute flow filed for different perforated sheet angle

\subsubsection{Influence of perforated sheet axial position}

In order to investigate the influence of the perforated sheet axial position on the aerodynamic performance, three different positions are now studied. As shown in the figure 15 , the distance from the right side of the perforated sheet to the right endwall is A. And A takes values of $2 \mathrm{~mm}, 6 \mathrm{~mm}$ and $10 \mathrm{~mm}$ respectively in this study.

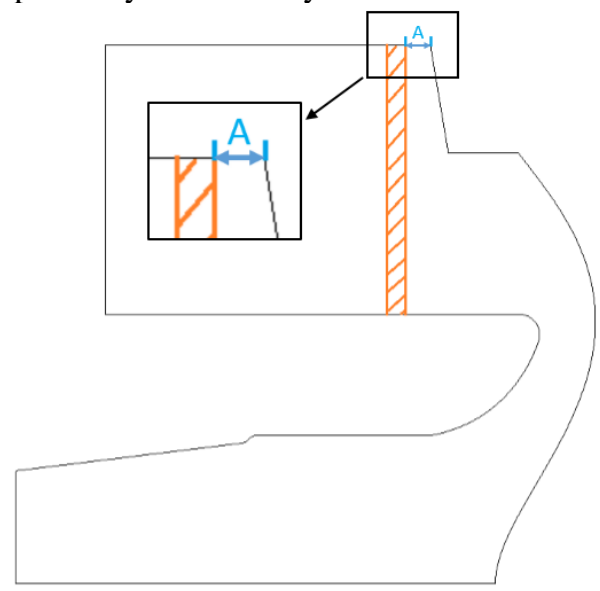

\section{Figure 15 Sketch map of perforated sheet axial position}

Figure 16 shows the dimensionless axial velocity at the exit of the volute with different axial position of perforated sheet. The black, green and red lines indicate that the distance between the right side of the perforated sheet to the right endwall is 2, 6 and $10 \mathrm{~mm}$. It is obvious from the value of Unevenness coefficient that the airflow uniformity of the $\mathrm{A}=6 \mathrm{~mm}$ volute is the best, the $\mathrm{A}=2 \mathrm{~mm}$ and $10 \mathrm{~mm}$ volute is the same poor, which indicates that there is an optimal axial direction to make the airflow uniformity the best.

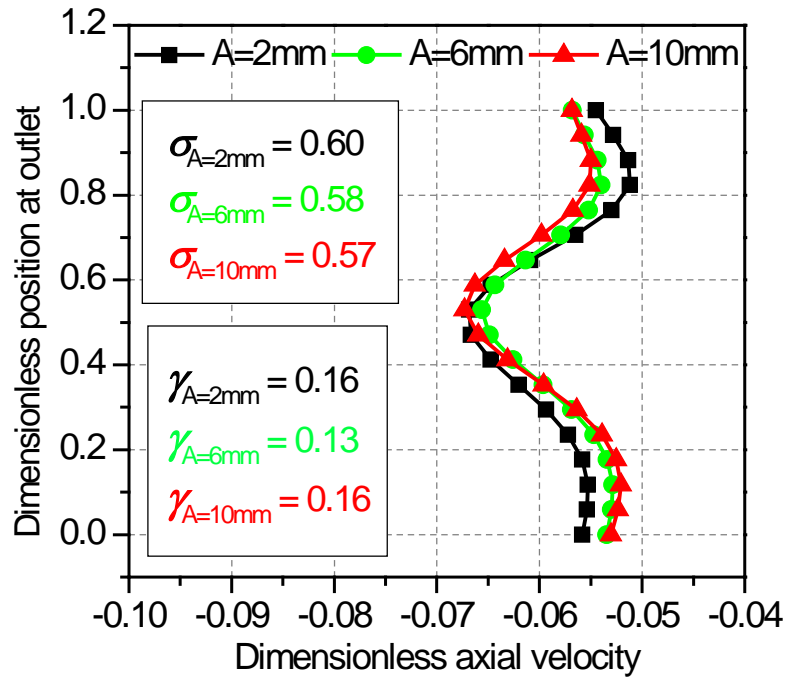

Figure 16 Axial velocity distribution at volute outlet
for different perforated sheet axial position

Fgure 17 shows the streamline diagram for different volutes. For the volute of $\mathrm{A}=2 \mathrm{~mm}$, since the distance between the perforated sheet and the right endwall is too close in high radius, the flow of air to the high radius is suppressed, resulting in a low velocity of the high radius airflow and a high velocity of the low radius airflow. For the $\mathrm{A}=10 \mathrm{~mm}$ volute, the distance between the perforated sheet and the right endwall is large, and there is no space blocking effect, the fluid flow is mainly affected by the Coanda effect, so that the airflow is attached to the wall surface and flows in a high radius direction. The airflow velocity is high at high radius and the velocity at low radius is small. The airflow of the $A=6 \mathrm{~mm}$ volute is more uniform under the combined of these two effects.

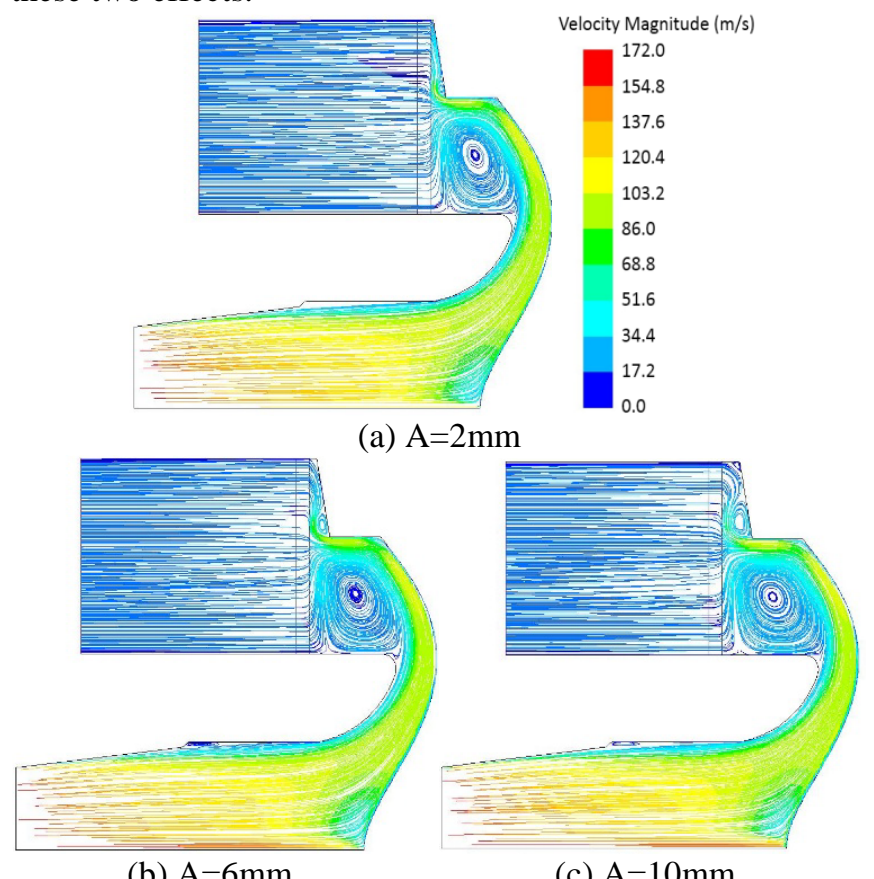

(b) $A=6 \mathrm{~mm}$

(c) $A=10 \mathrm{~mm}$

Figure 17 Comparison of volute flow filed for different perforated sheet axial position 


\subsection{Compare with Capstone C200}

Fgure 18 shows the airflow loss and evenness between Capstone's regenerative gas turbine volute and $A=6 \mathrm{~mm}$ volute in this paper. The geometry of C200 volute is similar to that of the volute in this paper. It can be see that the value of loss and unevenness coefficient is similar and the $A=6 \mathrm{~mm}$ volute is better. Using the method mentioned in paper to design axisymmetric volute which turn around $180 \mathrm{deg}$ is feasible.This also can verify the correctness of the calculation results in this paper.

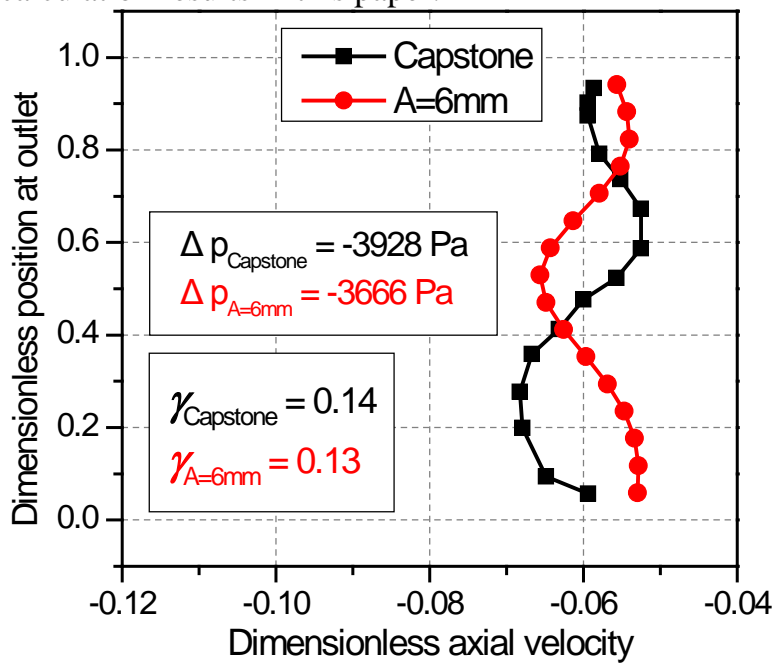

Figure 18 Parameter comparison between Capstone and $A=6 \mathrm{~mm}$ volute

\section{CONCLUSIONS}

Based on the design idea of coupling Coanda and flow separation mode to understand the flow mechanism of the axisymmetric volute which turn around $180 \mathrm{deg}$, and carry out parametric design to form design criteria to guide the design of this type of volute:

(1) Flow losses are mainly affected by profile of turn around section and the add of perforated sheet. The optimized profile of turn around can be found by optimization. Introducing the perforated sheet will increase loss a lot, but the angle and position of perforated sheet have little impact on it.

(2) Flow uniformity is mainly affected by profile and the perforated sheet. Two steps design will greatly improved the evenness coefficient. Introducing the perforated sheet will increase the evenness further more to meet the engineering requirements. When the space blocking effect is not considered, the perforated sheet angle has less influence on the airflow uniformity and aerodynamic loss. The perforated sheet position have a influence on evenness and it's controlled by Coanda effect and space blocking effect. We can use it to design the best perforated sheet to make airflow uniform.

(3)The fluid movement at high radius upstream of the perforated sheet is mainly determined by Coanda effect and space blocking effect. The Coanda effect causes the fluid attaching the wall to a high radius and increase the flow velocity in it. The space blocking effect caused by the small space makes the fluid moving toward the lower radius and thus increase the flow velocity in it.

\section{Nomenclature}

$\mathrm{A}=$ the distance from the right side of the perforated sheet to the right endwall

$m=$ mass flow

$p=$ static pressure

vertical $=$ perforated sheet vertical

l-oblique $=$ perforated sheet left oblique

r-oblique $=$ perforated sheet right oblique

$P_{\text {rec }}=$ total pressure recovery coefficient

$\sigma=$ loss coefficient

$v=$ velocity

$\rho=$ density

$\gamma=$ unevenness coefficient

$p^{*}=$ total pressure

$\bar{V}=$ area average velocity

Subscripts

in $=$ inlet of volute

$\max =$ bigger than average

out $=$ outlet of volute

ori $=$ original volute

opt $=$ optimization volute

seg $=$ two steps volute

$*=$ stagnation parameter

\section{REFERENCES}

[1] Zhou Z. H., Chen S. W., Lan Y. H. et al.(2014). Numerical investigation on the effect of tip clearance on a turbine stage flow-field and aerodynamic performance. Proceedings of the CSEE,2015(5).

[2] Xingsu L., Kunyuan P., Zuomin W.,(1981). Aerodynamic design and experimental study of marine gas turbine exhaust volutes. ASME 1981-GT-143.

[3] Cui Y. Q., Jiang H. D., (2003). Investigation of flow in a steam turbine exhaust hood with/without turbine exit conditions simulated. ASME J. Eng. Gas Turbines Power, 125(1): 292-299.

[4] Burton Z., Ingram G. L., Hogg S,. (2013). A literature review of low pressure steam turbine exhaust hood and diffuser studies. Journal of Engineering for Gas Turbines and Power, 135(6): 062001.

[5] Li Y., Yan Z., Cun L. F. et al., (2012). Numerical optimization on the volute configuration of a centrifugal fan via variable helical angle. Chinese Journal of Applied Mechanics. 29(4):398-403.

[6] Chen Z., Gu C. G., Shu X. W.,(2010). Shape optimum design for centrifugal compressor radial inlet based on CFD technique. Journal of Mechanical Engineering. 46(14):124-129.

[7] Huang E. D., Chu W. L., (2016). Optimization design of an asymmetry turbine exhaust hood. Journal of Propulsion Technology, 37(10):1839-1846.

[8] Wang X. Y., Gao C., Zhang Y. H., (2017). Combined design of flow adjusting device in diffuser of open- 
circuit wind tunnel with low speed. Journal of Machine Design. 2017(2).

[9] Shao D., Sun Z. G., Wu S. X., et al.(2018). Design exploration and experimental investigationfor a radial air intake system Journal of Propulsion Technology, 2018(9):1948-1954.

[10] Zhang X, Wu T.(2016). Effects of diffuser orifice plate on the performance of air-cooled steam condenser. Applied Thermal Engineering, 98: 179-188.

[11] Hao W. (2015). A numerical investigation of flow distribution in a new type of shell-and-tube heat exchanger. School of Energy Science and Engineering.

[12] Whitaker S. (1986). Flow in porous media I: A theoretical derivation of Darcy's law. Transport in porous media, 1(1): 3-25.

[13] McKay M D, Beckman R J, Conover W J.(1979). Comparison of three methods for selecting values of input variables in the analysis of output from a computer code. Technometrics, 21(2): 239-245.

[14] Tritton D J.(1977). Physical Fluid Dynamics Van Nostrand Reinhold. New York, 362.

[15] Islek A A.(2003). Optimization of micro compressor blades using CFD analysis and artificial intelligence. ASME FEDSM2003-45751.

[16] Capstone Turbine Corporation 2008, Advanced MicroTurbine System (AMTS) Final Technical Report, viewed 25 May 2019, https://docplayer.net/38147836Final-technical-report.html 